\title{
The Stereospecific Synthesis of the Rice Leaffolder Moth Sex Pheromone Components from 1,5-Cyclooctadiene ${ }^{\dagger}$
}

\author{
Sang Sook Kim and Yong Pyo Hong* \\ Department of Applied Chemistry, Andong National University. Andong 760-749, Korea. *E-mail: yphong@andong.ac.kr \\ Received February 11, 2011, Accepted March 1, 2011
}

Key Words : Rice leaffolder moth, Stereospecific synthesis, Pheromone, Coupling

The rice leaffolder moth, Cnaphalocrocis medinalis, is widely distributed in humid tropical to temperate countries of Asia, Oceania, and Africa. ${ }^{1}$ It is an important leaf feeding pest of rice. It's known that one leaf folder consumes 6 to 7 leaves during larval stage. Recently, it has become widespread throughout the major rice growing regions of Asia and become serious pests. ${ }^{2}$ The synthetic sex pheromone ${ }^{3}$ may not only be an effective monitoring tool for timing insecticide application, but also a possible control agent. Previously, two compounds, (Z)-11-hexadecenyl acetate (1, Z11-16:Ac) and (Z)-13-octadecenyl acetate (6, Z13-18:Ac), were identified as the female sex pheromone of the rice leaffolder moth and field tested in India and Philippines (Figure 1). ${ }^{4}$

On the other hand, additional four compounds, (Z)-11octadecen-1-ol (2, Z11-18:OH), (Z)-11-octadecenal (3, Z-1118:Ald), (Z)-13-octadecen-1-ol (4, Z13-18:OH), and (Z)-13octadecenal (5, Z13-18:Ald) were identified in Japan. ${ }^{5}$

In order to control the pest eco-friendly the obtention of each pheromone component is essential. A new method is described for the stereospecific syntheses of six sex pheromone components $\mathbf{1}$ to $\mathbf{6}$ for the rice leaffolder moth. The crucial synthetic step for the compounds is the introduction of pure (Z)-double bond in the molecules. The starting material, cis-1,8-oct-4-en-diol (7) consisting of $100 \%(Z)$ configuration was stereospecifically prepared from readily available 1,5-cyclooctadiene by known synthetic method (Scheme 1). ${ }^{6}$

The diol 7 was monoprotected with dihydropyran (DHP $)^{7}$ and then, tosylated to give compound $\mathbf{9}$. The Grignard reaction to the tosylate with methylmagnesium chloride or $n$-propylmagnesium chloride gave each coupling products $\mathbf{1 0}$ or $\mathbf{1 1}$ in high yields (Scheme 2). Continuously, deprotection ${ }^{6 a}$ and

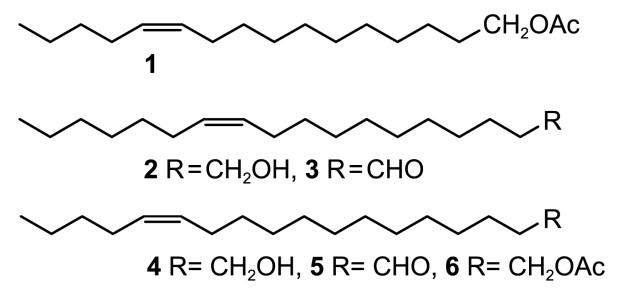

Figure 1. Six components of rice leaffolder moth sex pheromone.

"This paper is dedicated to Professor Eun Lee on the occasion of his honourable retirement. then, tosylation ${ }^{8}$ of the THP ethers produced the intermediates 14 and $\mathbf{1 5}$.

On the other hand, bromides $\mathbf{1 6}$ and $\mathbf{1 7}$ were easily prepared from the corresponding diols by monobromination and



Scheme 1. Synthesis of $c i$ s-1,8-oct-4-en-diol (7).

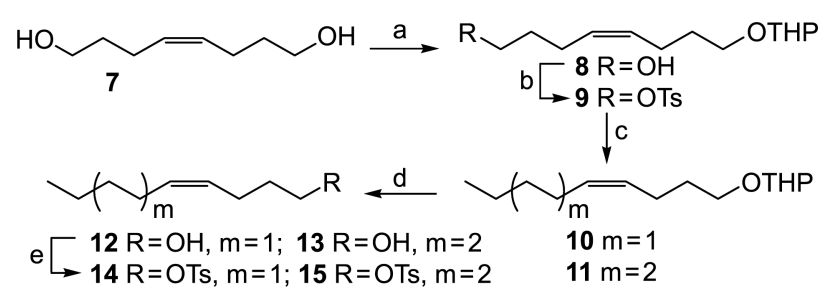

Scheme 2. Reagents and conditions: (a) DHP, PPTS, THF, 66\% (b) p-TsCl, pyridine, $89 \%$ (c) $\mathrm{CH}_{3} \mathrm{MgCl}, \mathrm{Li}_{2} \mathrm{CuCl}_{4}, \mathrm{THF}, 88 \%$ for $\mathbf{1 0}$; $n-\mathrm{C}_{3} \mathrm{H}_{7} \mathrm{MgCl}, 91 \%$ for 11 (d) PPTS, $\mathrm{MeOH}, 95 \%$ for $\mathbf{1 2} ; 99 \%$ for 13 (e) $p$-TsCl, pyridine, $78 \%$ for $14 ; 64 \%$ for 15 .

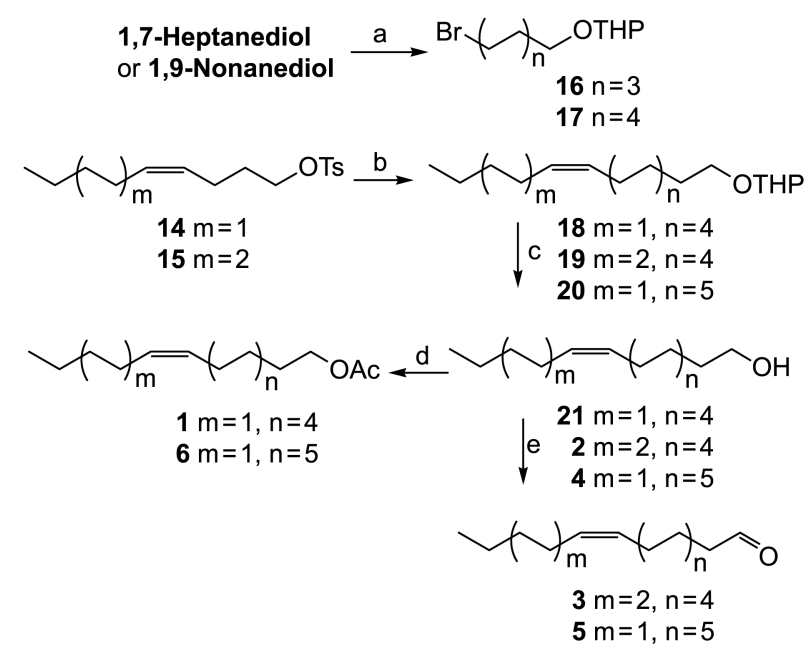

Scheme 3. Reagents and conditions: (a) HBr, benzene; DHP, PPTS, $\mathrm{CH}_{2} \mathrm{Cl}_{2}, 77 \%$ for $\mathbf{1 6} ; 74 \%$ for $\mathbf{1 7}$ (b) $\mathbf{1 6}$ or $\mathbf{1 7}, \mathrm{Mg}, \mathrm{Li}_{2} \mathrm{CuCl}_{4}, \mathrm{THF}$, $93 \%$ for $\mathbf{1 8} ; \mathbf{7 1} \%$ for $\mathbf{1 9} ; \mathbf{7 3} \%$ for $\mathbf{2 0}$ (c) PPTS, $\mathrm{EtOH}, 92 \%$ for $\mathbf{2 1}$; $88 \%$ for $2 ; 85 \%$ for 4 (d) $\mathrm{Ac}_{2} \mathrm{O}$, pyridine, $97 \%$ for $1 ; 96 \%$ for 6 (e) PCC, $\mathrm{CH}_{2} \mathrm{Cl}_{2}, 69 \%$ for $3 ; 75 \%$ for 5 . 
then, protection (Scheme 3). The second Grignard coupling reaction of the tosylates $\mathbf{1 4}$ or $\mathbf{1 5}$ with $\mathbf{1 6}$ or $\mathbf{1 7}$ gave the corresponding intermediates 18, 19, or 20 (Scheme 3). Deprotection and then, acetylation of $\mathbf{1 8}$ led to pheromone component Z11-16:Ac (1). Deprotection of 19 gave Z1118:OH (2), which led to Z11-18:Ald (3) by oxidation. On the other hand, deprotection of $\mathbf{2 0}$ produced pheromone component Z13-18:OH (4). Continuously, oxidation or acetylation of the alcohol 4 led each to pheromone components Z1318:Ald (5) or Z13-18:Ac (6).

In conclusion, the synthetic sex pheromone may not only be an effective monitoring tool for timing insecticide application, but also a possible control agent. In order to control the pest eco-friendly the obtention of each pheromone component is essential. Stereospecifically pure six sex pheromone components for the rice leaffolder moth were synthesized from readily available 1,5-cyclooctadiene.

\section{Experimental Section}

Genenral Techniques. IR spectra were recorded on a Jasco FT/IR 460 Plus, and NMR spectra were recorded on a Avance Digital $400 \mathrm{MHz}$ Spectrometer. All reactions were monitored by thin layer chromatography carried out on $0.25 \mathrm{~mm}$ E. Merck silica gel plates (60F-254) under UV light. All new compounds were identified by spectroscopic methods.

(Z)-8-(Tetrahydro-2H-pyran-2-yloxy)oct-4-enyl 4-methylbenzensulfonate (9). To a solution of compound 8 (1.00 g, $4.4 \mathrm{mmol})$ in pyridine $(30 \mathrm{~mL})$ was added $p$-toluenesulfonyl chloride $(1.25 \mathrm{~g}, 6.6 \mathrm{mmol})$ and stirred at room temperature for $4 \mathrm{~h}$. The reaction mixture was diluted with ethyl acetate and washed with cold $6 \mathrm{~N} \mathrm{HCl}$. The organic layer was dried $\left(\mathrm{Na}_{2} \mathrm{SO}_{4}\right)$ and evaporated in vacuo. The residue was purified by column chromatography (silica gel, 33\% ethyl acetate in hexane) to provide $9(1.50 \mathrm{~g}, 89 \%)$. IR (KBr) 3005, 2941, 2868, 1599, 1454, 1361, $1176 \mathrm{~cm}^{-1}$; ${ }^{1} \mathrm{H}$ NMR $(400 \mathrm{MHz}$, $\left.\mathrm{CDCl}_{3}\right) \delta 7.78(\mathrm{~d}, J=8.4 \mathrm{~Hz}, 2 \mathrm{H}), 7.34(\mathrm{~d}, J=8.4 \mathrm{~Hz}, 2 \mathrm{H})$, $5.42-5.35$ (m, $1 \mathrm{H}), 5.28-5.21 \mathrm{~m}, 1 \mathrm{H}), 4.55(\mathrm{dd}, J=4.4,2.8 \mathrm{~Hz}$, $1 \mathrm{H}), 4.01(\mathrm{t}, J=6.4 \mathrm{~Hz}, 2 \mathrm{H}), 3.88-3.83(\mathrm{~m}, 1 \mathrm{H}), 3.74-3.68(\mathrm{~m}$, $1 \mathrm{H}), 3.52-3.46(\mathrm{~m}, 1 \mathrm{H}), 3.38-3.32(\mathrm{~m}, 1 \mathrm{H}), 2.44$ (s, 3H), 2.09$2.01(\mathrm{~m}, 4 \mathrm{H}), 1.85-1.48(\mathrm{~m}, 10 \mathrm{H}) ;{ }^{13} \mathrm{C}$ NMR $(100 \mathrm{MHz}$, DMSO- $\left.d_{6}\right) \delta 144.7,133.1,130.8,129.8,127.9,127.8,98.9$, 70.0, 66.8, 62.4, 30.7, 29.6, 28.8, 25.4, 23.8, 23.0, 21.6, 19.7 .

(Z)-Non-4-en-1-ol (12). To a solution of 9 (6.00 g, 15.7 mmol) in dry THF $(12 \mathrm{~mL})$ was added $\mathrm{CH}_{3} \mathrm{MgCl}(13.6 \mathrm{~mL}$ of $3 \mathrm{M}, 40.8 \mathrm{mmol})$ at $-40{ }^{\circ} \mathrm{C}$ in the presence of $\mathrm{Li}_{2} \mathrm{CuCl}_{4}(0.1$ $\mathrm{M}$ in THF, $1.6 \mathrm{~mL}, 0.16 \mathrm{mmol})$. The reaction mixture was stirred at room temperature for $3 \mathrm{~h}$. After quenching the reaction with aqueous $\mathrm{NH}_{4} \mathrm{Cl}$ solution, the reaction mixture was diluted with $\mathrm{H}_{2} \mathrm{O}$ and extracted with ethyl ether. The organic layer was dried $\left(\mathrm{Na}_{2} \mathrm{SO}_{4}\right)$ and evaporated in vacuo. The residue was purified by column chromatography (silica gel, $10 \%$ ethyl acetate in hexane) to give the THP ether $\mathbf{1 0}$ (3.14 g, 88\%). Continuously, ether 10 (3.00 g, $13.2 \mathrm{mmol}$ ) was deprotected with PPTS at $80^{\circ} \mathrm{C}$ in $\mathrm{MeOH}$ to give alcohol $12(1.80 \mathrm{~g}, 95 \%)$ after purification. IR (KBr) 3334, 3005, 2928, $2860 \mathrm{~cm}^{-1}$; ${ }^{1} \mathrm{H}$ NMR (400 MHz, $\mathrm{CDCl}_{3}$ ) $\delta$ 5.43-5.33 $(\mathrm{m}, 2 \mathrm{H}), 3.65(\mathrm{t}, J=6.4 \mathrm{~Hz}, 2 \mathrm{H}), 2.17-2.10(\mathrm{~m}, 2 \mathrm{H}), 2.06-$ $2.01(\mathrm{~m}, 2 \mathrm{H}), 1.66-1.59(\mathrm{~m}, 2 \mathrm{H}), 1.35-1.26(\mathrm{~m}, 4 \mathrm{H}), 0.89(\mathrm{t}$, $J=8.4 \mathrm{~Hz}, 3 \mathrm{H}) ;{ }^{13} \mathrm{C} \mathrm{NMR}\left(100 \mathrm{MHz}, \mathrm{CDCl}_{3}\right) \delta 130.8$, 128.8, 62.6, 32.6, 31.9, 26.9, 23.6, 22.3, 14.0.

(Z)-Undec-4-en-1-ol (13). Prepared in 90\% yield (two step) in same method as that described for 12 except using $n$ $\mathrm{C}_{3} \mathrm{H}_{7} \mathrm{MgCl}$ instead of $\mathrm{CH}_{3} \mathrm{MgCl}$. IR ( $\mathrm{KBr}$ ) 3328, 3005, 2926, $2856 \mathrm{~cm}^{-1}$; ${ }^{1} \mathrm{H}$ NMR (400 MHz, $\mathrm{CDCl}_{3}$ ) $\delta$ 5.43-5.32 (m, 2H), 3.65 (t, $J=6.4 \mathrm{~Hz}, 2 \mathrm{H}), 2.14-2.09$ (m, 2H), 2.062.00 (m, 2H), 1.66-1.59 (m, 2H), 1.35-1.28 (m, 8H), 0.87 (t, $J$ $=7.4 \mathrm{~Hz}, 3 \mathrm{H}) ;{ }^{13} \mathrm{C} \mathrm{NMR}\left(100 \mathrm{MHz}, \mathrm{CDCl}_{3}\right) \delta 130.8,128.8$, 62.6, 32.6, 31.7, 29.6, 29.0, 27.2, 23.6, 22.6, 14.1.

(Z)-Hexadec-11-en-1-ol (21). To a solution of the tosylate $14(1.80 \mathrm{~g}, 6.1 \mathrm{mmol})$ in dry THF $(10 \mathrm{~mL})$ was added Grignard reagent prepared from $\mathrm{Mg}(0.56 \mathrm{~g}, 23.0 \mathrm{mmol})$ and bromide $16(5.37 \mathrm{~g}, 10.2 \mathrm{mmol})$ in THF $(5 \mathrm{~mL})$ at $-40^{\circ} \mathrm{C}$ in the presence of $\mathrm{Li}_{2} \mathrm{CuCl}_{4}(0.1 \mathrm{M}$ in THF, $0.64 \mathrm{~mL}, 0.064$ $\mathrm{mmol})$. The reaction mixture was stirred at room temperature for $1.5 \mathrm{~h}$. After quenching the reaction with aqueous $\mathrm{NH}_{4} \mathrm{Cl}$, the reaction mixture was diluted with $\mathrm{H}_{2} \mathrm{O}$ and extracted with ethyl ether. The organic layer was dried $\left(\mathrm{Na}_{2} \mathrm{SO}_{4}\right)$ and evaporated in vacuo. The residue was purified by column chromatography (silica gel, $10 \%$ ethyl acetate in hexane) to give the THP ether 18 (1.83 g, 93\%). Continuously, ether 18 $(1.97 \mathrm{~g}, 6.1 \mathrm{mmol})$ was deprotected with PPTS at $80{ }^{\circ} \mathrm{C}$ in EtOH to give the alcohol $21(1.34 \mathrm{~g}, 92 \%)$ after purification. IR (KBr) 3332, 3004, 2926, $2854 \mathrm{~cm}^{-1}$; ${ }^{1} \mathrm{H}$ NMR (400 MHz, $\left.\mathrm{CDCl}_{3}\right) \delta 5.38-5.30(\mathrm{~m}, 2 \mathrm{H}), 3.63(\mathrm{t}, J=6.4 \mathrm{~Hz}, 2 \mathrm{H}), 2.04-$ $1.98(\mathrm{~m}, 4 \mathrm{H}), 1.59-1.52(\mathrm{~m}, 2 \mathrm{H}), 1.36-1.25(\mathrm{~m}, 18 \mathrm{H}), 0.89(\mathrm{t}$, $J=8.4 \mathrm{~Hz}, 3 \mathrm{H}) ;{ }^{13} \mathrm{C} \mathrm{NMR}\left(100 \mathrm{MHz}, \mathrm{CDCl}_{3}\right) \delta 129.9,129.8$, 63.0, 32.8, 31.9, 29.7, 29.6, 29.5, 29.4, 29.3, 29.1, 27.2, 26.9, 25.7, 22.3, 14.0.

(Z)-Octadec-11-en-1-ol (2). Prepared in 62\% yield (two steps) in same method as that described for 21 except using the tolylate 15 instead of 14. IR (KBr) 3327, 3004, 2926, 2854 $\mathrm{cm}^{-1} ;{ }^{1} \mathrm{H}$ NMR $\left(400 \mathrm{MHz}, \mathrm{CDCl}_{3}\right) \delta$ 5.38-5.32 (m, 2H), 3.64 $(\mathrm{t}, J=6.4 \mathrm{~Hz}, 2 \mathrm{H}), 2.03-1.98(\mathrm{~m}, 2 \mathrm{H}), 1.60-1.53(\mathrm{~m}, 2 \mathrm{H})$, $1.37-1.23(\mathrm{~m}, 24 \mathrm{H}), 0.88(\mathrm{t}, J=7.2 \mathrm{~Hz}, 3 \mathrm{H}) ;{ }^{13} \mathrm{C} \mathrm{NMR}(100$ $\left.\mathrm{MHz}, \mathrm{CDCl}_{3}\right) \delta 129.9,129.8,63.1,32.8,31.8,31.7,29.8$, 29.7, 29.6, 29.5, 29.4, 29.3, 29.1, 29.0, 27.2, 25.7, 22.6, 14.1 .

(Z)-Octadec-13-en-1-ol (4). Prepared in 62\% yield (two steps) in same method as that described for 21 except using the bromide 17 instead of 16. IR (KBr) 3327, 3004, 2926, $2854 \mathrm{~cm}^{-1}$; ${ }^{1} \mathrm{H}$ NMR $\left(400 \mathrm{MHz}, \mathrm{CDCl}_{3}\right) \delta 5.38-5.31(\mathrm{~m}, 2 \mathrm{H})$, $3.63(\mathrm{t}, J=6.4 \mathrm{~Hz}, 2 \mathrm{H}), 2.04-1.99(\mathrm{~m}, 4 \mathrm{H}), 1.59-1.52(\mathrm{~m}$, $2 \mathrm{H}), 1.38-1.24(\mathrm{~m}, 22 \mathrm{H}), 0.89(\mathrm{t}, J=7.4 \mathrm{~Hz}, 3 \mathrm{H}) ;{ }^{13} \mathrm{C} \mathrm{NMR}$ $\left(100 \mathrm{MHz}, \mathrm{CDCl}_{3}\right) \delta 129.9,129.8,63.1,32.8,32.0,31.9$, 29.8, 29.6(3), 29.5, 29.4, 29.3, 27.2, 26.9, 25.7, 22.3, 14.0.

(Z)-Hexadec-11-enyl acetate (1). To a solution of alcohol $21(0.79 \mathrm{~g}, 3.3 \mathrm{mmol})$ in pyridine $(3.0 \mathrm{~mL})$ was added acetic anhydride $(0.62 \mathrm{~mL}, 6.6 \mathrm{mmol})$ and stirred at $0{ }^{\circ} \mathrm{C}$ for $3 \mathrm{~h}$. The reaction mixture was diluted with ice water and extracted with ethyl ether. The organic layer was washed with saturated $\mathrm{NaHCO}_{3}$ solution, water, saline, and then evaporated in vacuo. The residue was purified by column chromatography (silica gel, 25\% ethyl ether in hexane) to provide acetate $\mathbf{1}$ (0.90 g, 97\%). IR (KBr) 3004, 2926, 2855, 1743, 1238, 1038 
$\mathrm{cm}^{-1},{ }^{1} \mathrm{H}$ NMR (400 MHz, $\mathrm{CDCl}_{3}$ ) $\delta$ 5.32-5.24 (m, 2H), 3.98 $(\mathrm{t}, J=6.8 \mathrm{~Hz}, 2 \mathrm{H}), 1.98(\mathrm{~s}, 3 \mathrm{H}), 1.98-1.92(\mathrm{~m}, 4 \mathrm{H}), 1.56-1.51$ $(\mathrm{m}, 2 \mathrm{H}), 1.29-1.18(\mathrm{~m}, 18 \mathrm{H}), 0.83(\mathrm{t}, J=7.0 \mathrm{~Hz}, 3 \mathrm{H}) ;{ }^{13} \mathrm{C} \mathrm{NMR}$ $\left(100 \mathrm{MHz}, \mathrm{CDCl}_{3}\right) \delta 171.2,129.8$ (2), 64.6, 31.9, 29.7, 29.5 (2), 29.3, 29.2, 29.1, 28.6, 27.2, 26.9, 25.7, 22.3, 21.0, 14.0.

(Z)-Octadec-13-enyl acetate (6): Prepared in 96\% yield in the same method as that described for 1 except using the alcohol 4 instead of 21. IR (KBr) 3005, 2925, 2854, 1743, $1239,1039 \mathrm{~cm}^{-1} ;{ }^{1} \mathrm{H}$ NMR (400 MHz, $\left.\mathrm{CDCl}_{3}\right) \delta 5.32-5.24$ $(\mathrm{m}, 2 \mathrm{H}), 3.98(\mathrm{t}, J=6.8 \mathrm{~Hz}, 2 \mathrm{H}), 1.98(\mathrm{~s}, 3 \mathrm{H}), 1.99-1.92(\mathrm{~m}$, $4 \mathrm{H}), 1.58-1.51(\mathrm{~m}, 2 \mathrm{H}), 1.28-1.18(\mathrm{~m}, 22 \mathrm{H}), 0.83(\mathrm{t}, J=7.0$ $\mathrm{Hz}, 3 \mathrm{H}) ;{ }^{13} \mathrm{C} \mathrm{NMR}\left(100 \mathrm{MHz}, \mathrm{CDCl}_{3}\right) \delta 171.3,129.9,129.8$, 64.7, 31.9, 30.9, 29.8, 29.6 (2), 29.5 (2), 29.3, 29.2, 28.6, 27.2, 26.9, 25.9, 22.3, 21.0, 14.0.

(Z)-Octadec-11-enal (3): To a solution of alcohol 2 (0.50 g, $1.9 \mathrm{mmol})$ in dry dichloromethane $(8 \mathrm{~mL})$ was added PCC $(0.60 \mathrm{~g}, 2.8 \mathrm{mmol})$ at room temperature. After $3 \mathrm{~h}$ stirring, the reaction was quenched with a few drop of EtOH, and the reaction mixture was filtered with celite. After evaporating solvent, the residue was purified by column chromatography (silica gel, $10 \%$ ethyl acetate in hexane) to give aldehyde $\mathbf{3}$ (0.34 g, 69\%). IR (KBr) 3005, 2925, 2854, 2713, $1729 \mathrm{~cm}^{-1}$; ${ }^{1} \mathrm{H}$ NMR (400 MHz, $\left.\mathrm{CDCl}_{3}\right) \delta 9.76(\mathrm{t}, J=2.0 \mathrm{~Hz}, 1 \mathrm{H}), 5.38-$ $5.30(\mathrm{~m}, 2 \mathrm{H}), 2.42(\mathrm{td}, J=7.6,2.0 \mathrm{~Hz}, 2 \mathrm{H}), 2.03-1.98(\mathrm{~m}$, $4 \mathrm{H}), 1.66-1.59$ (m, 2H), 1.32-1.18 (m, 20H), 0.88 (t, $J=7.2$ $\mathrm{Hz}, 3 \mathrm{H}) ;{ }^{13} \mathrm{C} \mathrm{NMR}\left(100 \mathrm{MHz}, \mathrm{CDCl}_{3}\right) \delta 203.1,129.9,129.8$, 43.9, 31.8, 29.7, 29.5, 29.4, 29.3 (2), 29.2, 29.1, 29.0, 27.2 (2), 22.7, 22.1, 14.1

(Z)-Octadec-13-enal (5): Prepared in 75\% yield in the same method as that described for 3 except using the alcohol 4 instead of 2. IR (KBr) 3004, 2925, 2854, 2713, $1730 \mathrm{~cm}^{-1}$; ${ }^{1} \mathrm{H}$ NMR (400 MHz, $\left.\mathrm{CDCl}_{3}\right) \delta 9.76(\mathrm{t}, J=2.0 \mathrm{~Hz}, 1 \mathrm{H}), 5.38-$ $5.30(\mathrm{~m}, 2 \mathrm{H}), 2.41(\mathrm{td}, J=7.6,2.0 \mathrm{~Hz}, 2 \mathrm{H}), 2.01-1.96(\mathrm{~m}$,
$4 \mathrm{H}), 1.66-1.58(\mathrm{~m}, 2 \mathrm{H}), 1.35-1.23(\mathrm{~m}, 20 \mathrm{H}), 0.88(\mathrm{t}, J=7.2$ $\mathrm{Hz}, 3 \mathrm{H}) ;{ }^{13} \mathrm{C}$ NMR $\left(100 \mathrm{MHz}, \mathrm{CDCl}_{3}\right) \delta 203.1,129.9,129.8$, 43.9, 31.9, 29.7, 29.6 (2), 29.5, 29.4, 29.3 (2), 29.1, 27.2, $26.9,22.3,22.1,14.0$.

Acknowledgments. This work was supported by the research fund of Andong Nationl University 2009.

\section{References}

1. Sakai, K.; Ikeda, Y.; Sumeshima, J. Appl. Ent. 1942, 4, 1.

2. (a) Khan, Z. R.; Barrion, A. T.; Litsinger, J. A.; Castilla, N. P.; Joshi, R. C. Insect Science and its Application 1988, 9, 129. (b) Rani, W. B.; Amutha, R.; Muthulakshmi, M.; Indira, K.; Mareeswari, P. Res. J. Agric. \& Biol. Sci. 2007, 3, 394.

3. Kang, S.-K.; Moon, B.-H.; Lee, J.-O.; Goh, H.-G. Bull. Kor. Chem. Soc. 1985, 6, 228

4. (a) Ganeswara, R.; Krishnaiah, K.; Hall, D. R.; Cork, A.; Reddy, D. R. Indian Journal of Plant Protection 1993, 21, 228. (b) Ganeswara, R. A.; Reddy, D. D. R.; Krishnaiah, K.; Beever, P. S.; Cork, A.; Hall, D. R. Entomologia Experimentalis et Applicata 1995, 74, 195. (c) Ramachandran, R.; Caballero, P.; Khan, Z. R. Japanese Journal of Applied Entomology and Zoology 1990, 25, 26.

5. Kawazu, K.; Hasegawa, J.; Honda, H.; Ishikawa, Y.; Wakamura, S.; Sugie, H.; Kamiwada, H.; Kamimura, T.; Yoshiyasa, Y.; Tatsuki, S. Entomologia Experimentalis et Applicata 2000, 96, 103.

6. (a) Raederstorff, D. A.; Shu, Y. L.; Thompson, J. E.; Djerassi, C. J. Org. Chem. 1987, 52, 2337. (b) Nagarkatti, J. P.; Ashley, K. R. Tetrahedron Lett. 1973, 14, 4599. (c) Tolstikov, G. A.; Odinokov, V. N.; Galeeva, R. I.; Bakeeva, R. S. Tetrahedron Lett. 1978, 19, 1857.

7. Khan, A. T.; Choudhury, L. H.; Ghosh, S. Tetrahedron Lett. 2004, 45,7891 .

8. Johnson, W. S.; Collins, J. C.; Pappo, R.; Rubin, M. B.; Kropp, P. J.; Johns, W. F.; Pike, J. E.; Bartmann, W. J. Am. Chem. Soc. 1963, 85,1409 . 